\title{
Discrete breathers in nonlinear lattices: Experimental detection in a Josephson array
}

\author{
E. Trías ${ }^{1}$, J. J. Mazo ${ }^{1,2}$ and T. P. Orlando ${ }^{1}$ \\ ${ }^{1}$ Department of Electrical Engineering and Computer Science, \\ Massachusetts Institute of Technology, Cambridge, Massachusetts 02139, \\ ${ }^{2}$ Departamento de Física de la Materia Condensada and ICMA \\ CSIC-Universidad de Zaragoza, E-50009 Zaragoza, Spain
}

(August 12, 2018)

\begin{abstract}
We present an experimental study of discrete breathers in an underdamped Josephson-junction array. Breathers exist under a range of dc current biases and temperatures, and are detected by measuring dc voltages. We find the maximum allowable bias current for the breather is proportional to the array depinning current while the minimum current seems to be related to a junction retrapping mechanism. We have observed that this latter instability leads to the formation of multi-site breather states in the array. We have also studied the domain of existence of the breather at different values of the array parameters by varying the temperature.
\end{abstract}

Discrete breathers are a new type of excitation in nonlinear lattices. They are characterized by an exponential localization of the energy. This localization does not occur in linear systems and it is different from Anderson localization, which is due to the presence of impurities. Thus, discrete breathers are also known as intrinsic localized modes.

Breathers have been proven to be generic solutions for the dynamics of nonlinear coupled oscillator systems [1.2 by the use of the novel mathematical technique of the anti-integrable limit [3]. They have been extensively studied [4 \&] and have been proposed to theoretically exist in diverse systems such as in spin wave modes of antiferromagnets [9], DNA denaturation [10], and the dynamics of Josephson-junction networks [11,12]. Also, they have been shown to be important in the dynamics of mechanical engineering systems [13,14]. Although a number of experiments have been proposed, discrete breathers have yet to be experimentally generated and measured.

In this Letter, we present, to our knowledge, the first experimental study of discrete breathers in a spatially extended system. We have designed and fabricated an underdamped Josephson-junction ladder which allows for the existence of breathers when biased by dc external currents. We have developed a method for exciting breathers and explored their existence domain and instability mechanisms with respects to the junction parameters and the applied current.

A Josephson junction consists of two superconducting leads separated by a thin insulating barrier. Due to the Josephson effect, it behaves as a solid-state nonlinear oscillator and is usually modeled by the same dynamical equations that govern the motion of a driven pendulum [15, 16]: $i=\ddot{\varphi}+\Gamma \dot{\varphi}+\sin \varphi$. The response of the junction to a current is measured by the voltage of the junction which is given by $v=\left(\Phi_{0} / 2 \pi\right) d \varphi / d t$. By coupling junctions it is possible to construct solid-state physical realizations of different models such as the Frenkel-

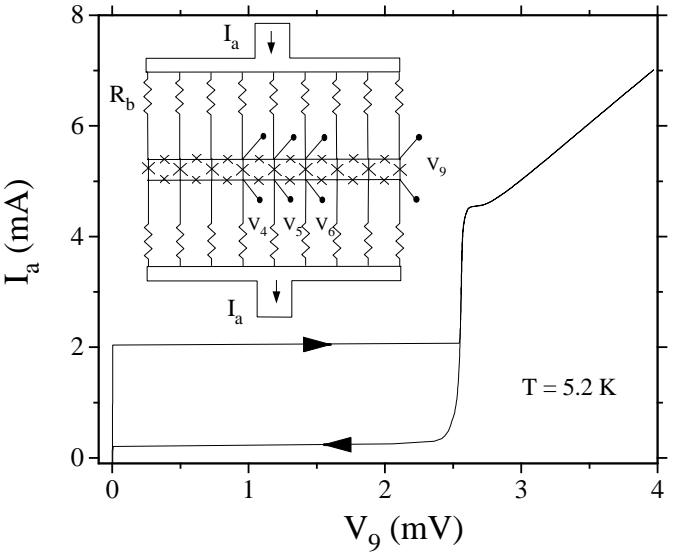

FIG. 1. Current-voltage characteristic of an anisotropic Josephson junction array. The hysteresis between the depinning current $\left(I_{d e p} \approx 2 \mathrm{~mA}\right)$ and the retrapping current $\left(I_{r} \approx 0.2 \mathrm{~mA}\right)$ is shown. Inset: Schematic of the anisotropic ladder array. Vertical junctions have four times the area of the horizontal ones. Current is injected through bias resistors, $R_{b}$, of $25 \Omega$. These resistors are larger than the vertical junction's normal state resistance of $5 \Omega$ to assure a more homogeneous current drive and are also larger then the horizontal's normal state resistance of $20 \Omega$ to minimize effects on their dynamics. There are voltage probes in the fourth, fifth, sixth and ninth vertical junctions to measure $V_{4}, V_{5}, V_{6}$ and $V_{9}$. The voltage probes can also be used to measure the top horizontal junctions in the middle which we denote as $V_{4 T}$ and $V_{5 T}$.

Kontorova 177 model for nonlinear dynamics and the 2D $\mathrm{XY}$ model 18 for phase transitions in condensed matter. Moreover, since the parameters, such as $\Gamma(T)$, vary with temperature, a range of parameter space can be studied easily with each sample.

The inset of Fig. 1 1 shows a schematic of the anisotropic ladder array. The junctions are fabricated using a Nb$\mathrm{Al}_{2} \mathrm{O}_{x}$ - Nb tri-layer technology with a critical current density of $1000 \mathrm{~A} / \mathrm{cm}^{2}$. The current is injected and extracted through bias resistors in order to distribute the current 
as uniformly as possible through the array. These resistors are large enough so as to minimize any deleterious effects on the dynamics. The anisotropy of the array is defined by $h$ as the ratio of areas of the horizontal to vertical junctions. In our arrays $h=1 / 4$ and $h=I_{c h} / I_{c v}=R_{v} / R_{h}=C_{h} / C_{v}$, and $\Gamma_{v}=\Gamma_{h}=\Gamma$. As shown in the schematic, we have placed voltage probes at various junctions in order to measure the voltages of both horizontal and vertical junctions.

In Fig. 11 we show a typical current-voltage, IV, characteristic of the array. As the applied current increases from zero we measure the average voltage of the 9 -th junction. The junction starts at a zero-voltage state and remains there until it reaches the array's depinning current $I_{d e p}$ at about $2 \mathrm{~mA}$. The depinning current can roughly be understood as the sum of the vertical junction's intrinsic critical current $I_{c v}$ and the small circulating Meissner current around the array. In a pendulum analogy, the critical current is equivalent to the critical torque, which is just sufficiently strong enough to force the pendulum to start rotating. When the current is larger the junction switches from zero-voltage state to the junction's superconducting gap voltage, $V_{g}$, which at this temperature is $2.5 \mathrm{mV}$. At this point all of the vertical junctions are said to be rotating and the array is in its "whirling state". One of the effects of this gap voltage is to substantially affect the junction's resistance, and thereby damping, in a complicated nonlinear way. The current can be further increased until the junction reaches its normal state and it behaves as a resistor, $R_{n}$, of $5 \Omega$. As the current decreases the junction returns to the gap voltage and then to its zero-voltage state at the retrapping current, $I_{r}$, of $\approx 0.2 \mathrm{~mA}$. The hysteresis loop between $I_{d e p}$ and $I_{r}$ is due to our underdamped junctions: the inertia causes the junctions to continue to rotate when the applied current is lowered from above its critical value.

It is this hysteresis loop that allows for the existence of breathers in the ladder with dc bias current. In this current range the zero-voltage $(V=0)$ and rotating $\left(V=V_{g}\right)$ solutions coexist. Then, a discrete breather in the ladder corresponds to when one vertical junction is rotating while the other vertical junctions librate. This solution is easy to conceive in the limit where the vertical junctions are imagined to be completely decoupled. However, whether a localized solution can exist in the ladder will be determined by the strength of the spatial coupling between vertical junctions. This coupling occurs through three mechanisms: flux quantization, self and mutual inductances of the meshes, and the horizontal junctions. Though the effective coupling is a complicated function of the array parameters, it is most strongly controlled by $h$. If the anisotropy $h$ is too large, then the vertical junctions will not support localized solutions that can be excited by dc currents. It has been determined from simulations of the system 12 that $h=1 / 4$ will allow for the existence of breathers in our ladders.

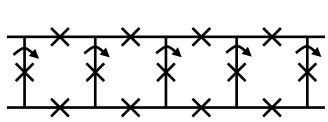

(a)

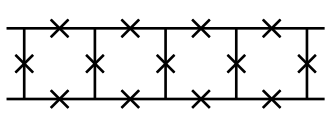

(b)

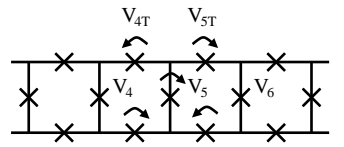

(c)

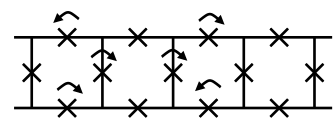

(d)
FIG. 2. Schematic of solutions in the array: (a) the whirling state where vertical junctions are rotating and horizontal ones librate; (b) the zero-voltage state where there are no rotating junctions; (c) a breather solution where the fifth vertical junction and the nearest horizontal neighbors rotate and the other vertical and horizontal junctions librate; (d) a multi-site breather solution where two vertical junctions rotate.

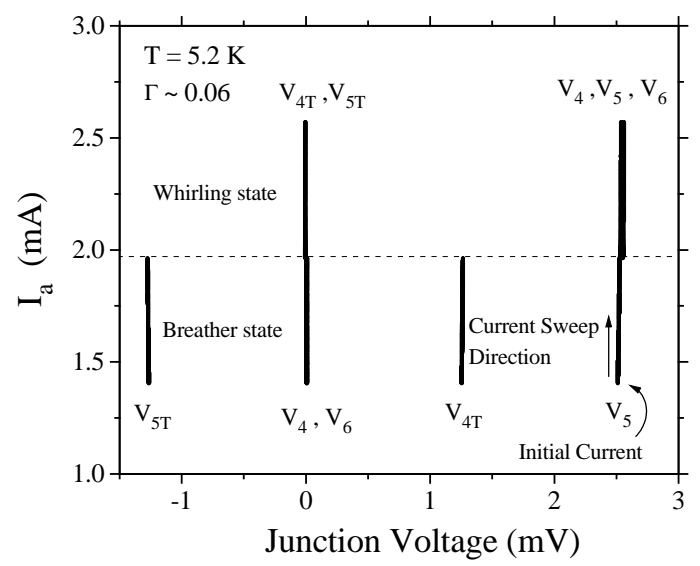

FIG. 3. Measured averaged voltages of five junctions in the center of array as the applied current is increased. We have biased the ladder at $1.4 \mathrm{~mA}$ and excited a breather as indicated in the text. Then the applied current is increased. There are two regions in the $\mathrm{IV}$. Below $\approx 2 \mathrm{~mA}$ we see the breather; above, the breather becomes unstable and the array switches to the whirling state.

Figure 2 shows some possible solutions for the states of our ladder. Graph (a) is the whirling state with every vertical junction rotating as indicated by the arrows. This is the state when all the junctions of the array have switched to the gap voltage. Graph (b) shows the zero voltage state with no rotating junctions. Graph (c) depicts a single-site breather solution. Here, one of the vertical and the horizontal neighboring junctions rotate. The horizontal junctions allow the vertical junction to rotate with a mean voltage without an overall increase of the stored magnetic energy. Graph (d) shows a twosited breather where two vertical junctions rotate. We have experimentally detected these types of localized solutions (c and d) by measuring the average dc voltage of the junctions as labeled in (c).

For our experiments, we have developed a simple reproducible method of exciting a breather: (i) bias the 
array uniformly to a current below depinning current; (ii) increase the current injected into the middle vertical junction [labeled $V_{5}$ in Fig. 2(c)] until its voltage switches to the gap; (iii) reduce this extra current in the middle junction to zero.

For example, to prepare the initial state in Fig. 3 we started by increasing the applied current to $1.4 \mathrm{~mA}$ which is below $I_{d e p}$. At this point the array is in the zero-voltage state. We then add an extra bias current to the middle junction (number 5) until it switches to the gap voltage of $2.5 \mathrm{mV}$ and then we reduce this extra bias to zero. In a sense we have prepared the initial conditions for the experiment. We can now increase the uniform applied current while simultaneously measuring the voltages of the vertical junctions $\left(V_{4}, V_{5}\right.$ and $\left.V_{6}\right)$ and the top two horizontal junctions, $V_{4 T}$ and $V_{5 T}$, as labeled in Fig. 2(c).

Figure 3 shows the result after we have excited the breather and we have increased the array current. Close to the initial current of $1.4 \mathrm{~mA}$ only the fifth vertical junction is at $V_{g}$ and both the fourth and sixth vertical junctions are in the zero-voltage state. This is the breather state shown in Fig. 2(c) and in essence the signature of the localized breather: a vertical junction is rotating while its neighboring vertical junctions do not rotate. We also see that both neighboring horizontal junctions have a voltage magnitude that is precisely half of this value $\left(V_{4 T}=-V_{5 T}=V_{5} / 2\right.$ and $\left.V_{4}=V_{6}=0\right)$. Both the magnitude and the sign can be understood by applying Kirchoff's voltage law to top-bottom voltage-symmetric solutions, as sketched in Fig. 2(c). The voltage of the top horizontal junction is equal to the negative of the bottom one. Since the voltage drops around the loop must be zero, the horizontal voltages must be half that of the active vertical junction voltage. As we increase the current the breather continues to exist until the applied current approaches $I_{+} \approx 2 \mathrm{~mA}$. At this point the horizontal junctions switch to a zero-voltage state while all of the vertical junctions switch to $V_{g}=2.5 \mathrm{mV}$. The array is now in its whirling state as drawn in Fig. 2(a) where $V_{4}=V_{6}=V_{5}=V_{g}$ and $V_{4 T}=V_{5 T}=0$.

If we excite the breather again but instead of increasing the applied current we decrease it, we measure curves typical of Fig. 4. As explained above, we prepare the array in an initial condition with a breather located in junction 5 at $1.4 \mathrm{~mA}$. We then decrease the applied current slowly. We start with the signature measurement of the breather: junction five is rotating at $V_{g}$ while $V_{4}$ and $V_{6}=0$. We also see that the horizontal junctions have the expected value of $V_{g} / 2$. As the current is decreased the breather persists until the array is biased at $0.8 \mathrm{~mA}$. The fourth vertical junction then switches to the gap voltage while $V_{4 T}$ switches to a zero voltage state. The resulting array state is sketched in Fig. 2(d) with $V_{4}=V_{5}=V_{g}$ while $V_{5 T}=V_{g} / 2$ and $V_{4 T}=V_{6}=0$. The single-site breather has destabilized by creating a two-site breather.

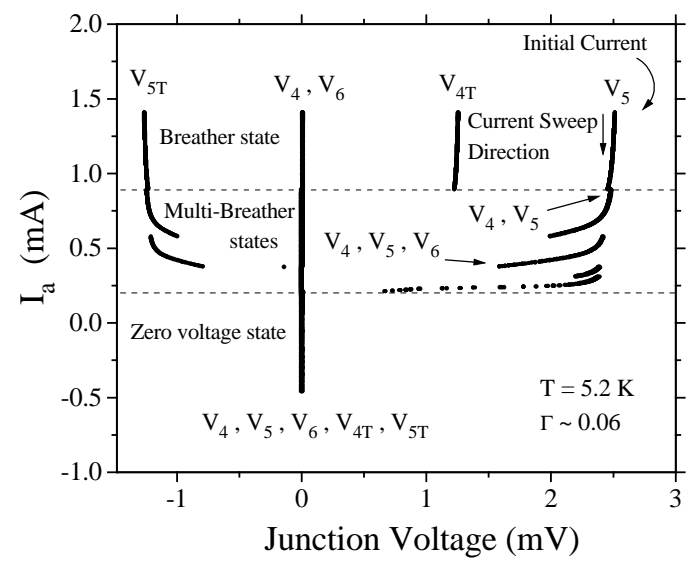

FIG. 4. Measured average voltages of five junctions in the center of array as the applied current is decreased. We have biased the ladder at $1.4 \mathrm{~mA}$ and excited a breather as indicated in the text. Then the applied current is decreased. There are three regions in the IV. The breather state becomes unstable at around $0.8 \mathrm{~mA}$ leading to a sequence of shifts at the gap voltage that are interpreted as multi-site breather states. The array reaches its zero-voltage state at about $0.2 \mathrm{~mA}$.

As the applied current is further decreased beyond the single-site breather instability at $\approx 0.8 \mathrm{~mA}$, the voltage of the fourth and fifth vertical junctions decreases but then suddenly jumps back to $V_{g}$. Then the voltage decreases again, and it again jumps back to $V_{g}$. This second shift corresponds to the sixth junction switching from the zero voltage state to the gap voltage. At this current bias, all of the three measured vertical voltages are rotating. There is a further jump of the voltage as the current decreases. Finally, at $0.2 \mathrm{~mA}$ all of the vertical junctions return to their zero-voltage state via a retrapping mechanism analogous to that of a single pendulum.

From these experiments and corroborating numerical simulations we speculate that this shifting of the voltage back to $V_{g}$ corresponds to at least one vertical junction switching from the zero-voltage state to the rotating state. The shapes of the IV curves in this multi-site breather regime are influenced by the redistribution of current when each vertical junction switches. This redistribution may also govern the evolution of the system after each transition to one of the other possible breather attractors in the phase space of the array. However, the exact nature of the selection process is not yet understood.

The above data was taken at a temperature of $5.2 \mathrm{~K}$. We found four current values of importance: the current when the array returns to the zero-voltage, $I_{r}$; the maximum zero-voltage state current, $I_{d e p}$; the maximum current the breather supports, $I_{+}$; and the minimum current $I_{-}$. By sweeping the temperature we can study how the current range in which our breather exists is affected by a change of the array parameters. 


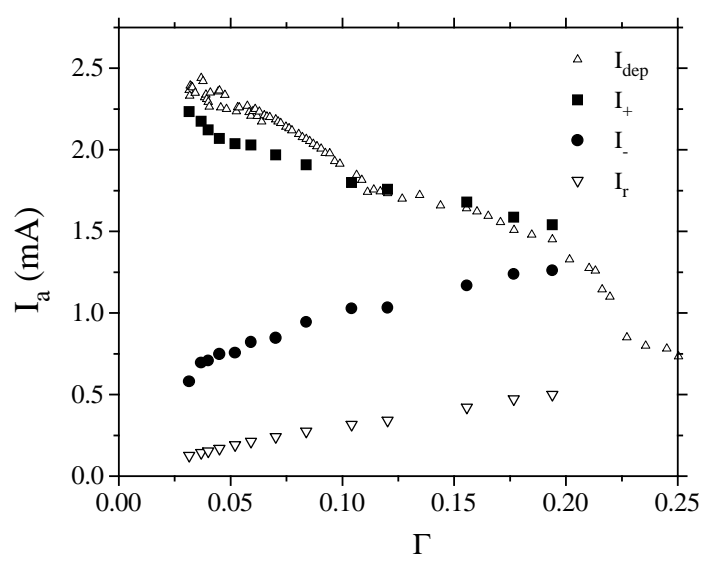

FIG. 5. Existence region of the breather in the current- $\Gamma$ plane. $\Gamma$ was varied by changing temperature. $I_{+}$is the maximum applied current the breather supports, while $I_{-}$is the minimum current.

Figure 5 shows the results of plotting the four special current values versus $\Gamma$. To calculate how the junction parameters vary with temperature we take $I_{c v}(0) R_{n}=$ $1.9 \mathrm{mV}$ and assume that the junction critical current follows the standard Ambegaokar-Baratoff dependence [19]. We estimate $\Gamma$ from $I_{r}$ by the relation $I_{r} / N I_{c v}=(4 / \pi) \Gamma$ [20], where $N$ is the number of vertical junctions in the array. The other relevant parameter is the dimensionless penetration depth, $\lambda_{\perp}=\Phi_{0} / 2 \pi L_{s} I_{c v}$, which measures the inductive coupling in the array. The loop inductance $L_{s}$ is estimated from numerical modeling of the circuit. By changing the temperature of the sample, we vary the $I_{c v}$ of the junction and hence change $\Gamma$ and $\lambda_{\perp}$. In this sample, the junction parameters can range from $0.031<\Gamma<0.61$ and $0.04<\lambda_{\perp}<0.43$ as the temperature varies from $4.2 \mathrm{~K}$ to $9.2 \mathrm{~K}$. In Fig. 5 , $\Gamma<0.2$ corresponds to $T<6.7 \mathrm{~K}$ and $\lambda_{\perp}<0.05$. At these low temperatures, there is a larger variation in $\Gamma$ because of the sensitive dependence of this parameter to the junction's resistance below the gap voltage.

As Fig. 5 shows, the maximum current supported by the breather, $I_{+}$, is almost equal to $I_{d e p}$. A simple circuit model gives some physical intuition. Junctions that are rotating have some effective resistance while junctions that are in the zero-voltage state have zero resistance. In our breather state, the center junction is rotating. Therefore, when we apply a current to our array the current will tend to flow around the rotating junction and through the outside junctions that are in the zero-voltage state. When these outside junctions reach their critical currents they will begin to rotate and the breather will disappear. In the simplest case, when we ignore any circulating Meissner currents, this model yields $I_{+} / N I_{c v}=(h+1) /(2 h+1)=0.8$. Since $I_{d e p}$ is roughly $N I_{c v}$, the depinning current is the upper bound for the applied current that the breather can support.
The instability mechanism that determines $I_{-}$in our experiments is more difficult to discern. We offer two suggestions that are due to the underdamped character of our system. One possibility is via a retrapping mechanism similar to that of a single junction. As the middle junction rotates, it reaches a point where the current drive is not sufficient to support the rotation and it destabilizes. This physical picture gives $I_{-} / N I_{c v}=(2 h+2)(4 / \pi) \Gamma$. So that for our parameters, $I_{-}$should be 2.5 times larger than $I_{r}$, as it is approximately in Fig. 5. A second possible instability mechanism consist of resonances between the characteristic frequencies of the breather and the lattice eigenmodes. The breather looses energy as it excites the eigenmodes. In our experiments, the breather always looses stability at voltages close to $V_{g}$. For our parameter range, $V_{g}$ is larger than the voltages for the lattice eigenmodes, thus our data seems to favor a retrapping mechanism. Lastly, we add that since $I_{d e p}$ and consequently $I_{+}$decreases with $\Gamma$, there also seems to be a critical damping where the breather will cease to exist. Experimentally we did not find a breather for $\Gamma>0.2$.

In summary, we have experimentally detected different breather and multi-site breather states in a superconducting Josephson ladder network. By varying the external current and temperature we have studied the domain of existence and the instability mechanisms of these localized solutions. In addition we have also found, but not discussed here, breathers which are not top-bottom voltage symmetric [12], in which only the top (bottom) horizontal junctions rotate while the bottom (top) junctions are in the zero-voltage state. These experiments are the first observations of discrete breathers and multi-site breathers in a condensed matter system.

This work was supported by NSF grant DMR-9610042 and DGES (PB95-0797). JJM thanks the Fulbright Commission and the MEC (Spain) for financial support. We thank S. H. Strogatz, A. E. Duwel, F. Falo, L. M. Floría, and P. J. Martínez for insightful discussions.

[1] R. S. MacKay and S. Aubry, Nonlinearity 7, 1623 (1994).

[2] J.-A. Sepulchre and R. S. MacKay, Nonlinearity 10, 679 (1997).

[3] S. Aubry and G. Abramovici, Physica D 43, 199 (1990).

[4] A. J. Sievers and S. Takeno, Phys. Rev. Lett. 61, 970 (1988).

[5] A. J. Sievers and J. B. Page, in Dynamical Properties of Solids VII, edited by G. K. Horton and A. A. Maradudin (North-Holand, Amsterdam, 1995), p. 137.

[6] S. Takeno and M. Peyrard, Physica D 92, 140 (1996).

[7] S. Aubry, Physica D 103, 201 (1997).

[8] S. Flach and C. R. Willis, Phys. Rep. 295, 181 (1998). 
[9] R. Lai and A. J. Sievers, Phys. Rev. Lett. 81, 1937 (1998).

[10] M. Peyrard, Europhys. Lett. 44, 271 (1998).

[11] L. M. Floría, J. L. Marín, P. J. Martínez, F. Falo and S. Aubry, Europhys. Lett. 36, 539 (1996).

[12] J. J. Mazo, E. Trías, and T. P. Orlando, to appear in Phys. Rev. B.

[13] J. Aubrecht, A. F. Vakakis, T.-C. Tsao, and J. Bentsman, Journal of Sound and Vibration 195, 629 (1996).

[14] A. F. Vakakis, A. N. Kounadis, and I. G. Raftoyiannis, Earthquake Eng. and Structural Dynamics 28, 21 (1999).

[15] S. H. Strogatz, Nonlinear dynamics and chaos (AddisonWesley, Massachusetts, 1994).

[16] The current that passes through the junction $i$ is normalized by the junction's intrinsic critical current $I_{c}(T)$. $\varphi$ is the gauge-invariant phase difference across the junction and $\Gamma$, the damping, is usually referred to as the Stewart-McCumber parameter $\beta_{c}=\Gamma^{-2}=2 \pi I_{c} C R^{2} / \Phi_{0}$ ( $C$ is the capacitance of the junction, $R$ is its resistance, and $\Phi_{0}$ is the flux quantum). Time is normalized by $\tau=\sqrt{\Phi_{0} C / 2 \pi I_{c}}$.

[17] S. Watanabe, S. H. Strogatz, H. S. J. van der Zant, and T. P. Orlando, Phys. Rev. Lett. 74, 379 (1995).

[18] D. J. Resnick, J. C. Garland, J. T. Boyd, S. Shoemaker, and R. S. Newrock, Phys. Rev. Lett. 47, 1542 (1981).

[19] V. Ambegaokar and A. Baratoff, Phys. Rev. Lett. 10, 486 (1963).

[20] J. Guckenheimer and P. Holmes, Nonlinear oscillations, dynamical systems, and bifurcations of vector fields (Springer-Verlag, New York, 1986). 